\title{
Relative Performance of Certain Meta Heuristics on Vehicle Routing Problem with Time Windows
}

\author{
Sandhya \\ M.M.E.C,Maharishi Markandeswar University, Ambala, India \\ Email: sandhya12bansal@gmail.com \\ V. Katiyar \\ M.M.E.C,Maharishi Markandeswar University, Ambala, India \\ Email: katiyarvk@mmumullana.org
}

\begin{abstract}
Solving Vehicle Routing Problem (VRP) and its variants arise in many real life distribution systems. Classical VRP can be described as the problem of finding minimum cost routes with identical vehicles having fixed capacity which starts from a depot and reaches a number of customers with known demands with the proviso that each route starts and ends at the depot and the demand of each customer does not exceed the vehicle capacity is met One of the generalizations of standard VRP is Vehicle Routing Problem with Time Windows (VRPTW) with added complexity of serving every customer within a specified time window. Since VRPTW is a NP hard meta heuristics have often been designed for solving it. In this paper we compare the performance of Simulated Annealing (SA), genetic Algorithm (GA) and Ant Colony Optimization (ACO) for solving VRPTW based on their performance using different parameters taking total travel distance as the objective to be minimized. The results indicate that ACO is in general slightly more efficient then SA and GA.
\end{abstract}

Index Terms - Vehicle Routing Problem, Time windows, Simulated Annealing, Genetic Algorithm, Ant Colony Optimization.

\section{INTRODUCTION}

In today's competitive environment it is necessary to save critical resources. Transportation is one of the major modes of consumption of energy as it is used for transportation of goods between different locations. Moreover it also contributes to the global warming by emitting $\mathrm{CO}_{2}$. Therefore best possible utilization of transportation systems is key to reduction in energy consumption as well as global warming. One of the important problem and widely studied in operation research that deals with efficient routing and scheduling of fleets is Vehicle Routing Problem (VRP).

A typical VRP can be described as problem of finding least cost route between depot and customers in such a way that each customer is visited once and only once by identical capacity vehicles such that total demand on each route does not exceed the vehicle capacity. The objective of the problem is to minimize the total traveled distance and the number of vehicles used so that both operational and maintaince cost is reduced.

The Vehicle Routing Problem with Time Windows (VRPTW) is variant of VRP with additional complexity of serving each customer within its time windows. VRPTW arises in many real life scenarios like bank, hot meal, perishable food deliveries etc. The time window of a customer, $i$, is represented by $\left[e_{i}, l_{i}\right]$ where $e_{i}$ and $l_{i}$ are the earliest and latest arrival time respectively. Service to a customer will be provided by a vehicle only if it arrives within this time period as shown in Fig 1 (a). A vehicle can arrive at customer location before its opening window but it has to wait till opening of window as shown in Fig 1 (b) but cannot after its closing window as shown in Fig 1 (c).
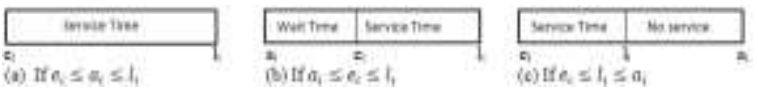

Fig. 1. (a)-(c) Time windows for customer $i$

VRP is generalization of famous combinatorial problem TSP with multiple vehicles. VRP is NP hard problem and it is one of the most difficult problem to solve in operation research. Many exact and meta heuristic approaches are applied to solve the problem but there exists no algorithm that guarantee to find optimal solution within a computational time. VRPTW is even more complex because of additional constraints. Even for instances with few customers and vehicles efficient routing is a difficult task. Solution space rapidly grows with a small increase in input size hence manual calculated routes are ineffective and human planner looks for automated planning tools to reduce the workload and time. Meta heuristics approaches which stand between exact and heuristic approaches are often tried for finding solutions to reach problems in reasonable time. Moreover they are also used for solving other problem too [26]. SA, GA, ACO, local searches, neural network, variable neighborhood search are some of the examples of meta heuristics. A review of application of neural network can be found in [27].

In this paper three basic meta heuristics namely Simulated Annealing (SA), Genetic (GA) and Ant Colony System (ACS) were implemented and analyzed 
using different parameters for different datasets by considering total travel distance as the primary objective to be minimized. The main contribution of this paper is that it provides a categorization of various meta heuristics for VRPTW and empirical analysis of three different meta heuristics on different parameters.

\section{LITERATURE REVIEW}

A state of the art in the field of VRPTW with heuristic approach is discussed in [1]. In this state of art overview of various methods along with their comparison on different parameters are described. Various heuristic techniques for solution construction and solution improvement phase are discussed in [2]. A survey on the progress and evolution of the problem statement is presented in [3]. In this paper various possible algorithms with their pros and cons are discussed. A taxonomy review encompassing all of the managerial, physical, theoretical disciplines are presented in [4]. A parallel mimetic algorithm for VRPTW is proposed in [5]. In this algorithm genetic and some local refinement procedure are executed in parallel to find solution. For improving the results a novel ran doming scheme is developed by which process communication takes place. A Particle Swarm Optimization algorithm is proposed for solving VRPTW in [6]. Furthermore the proposed algorithm is validated on a real world case study of Chlorine Capsule Distribution Company to the water reservoir in Tehran. A two phase hybrid meta heuristic for VRPTW is presented in [7]. In this firstly variable neighborhood search is applied to minimize the number of routes and then in second phase total travelled distance is minimized using tabu search algorithm. All exchange, all -2-opt, all cross exchange and all relocate and ejection chain operators are designed. A genetic algorithm using an optimized cross over operator is used in [8] to find optimal solution for VRPTW. VRPTW is solved using GA and PSO in [9]. Both single and hybridization of both meta heuristics were implemented. It is concluded that PSO perform better for clustered problems and GA is better for random customer distribution. A review of exact heuristics and meta heuristics methods for solving VRPTW is surveyed in [10]. Mathematical analysis of various meta heuristics is discussed in [11]. In their work authors had provided an overview of convergence and efficiency studies of meta heuristics and provided a frame work for analyzing meta heuristics in term of convergence and efficiency. Open challenge for future work is also provided. In [12] two meta heuristics named SA and iterated local search are applied to solve the problem. Both algorithms use constructive heuristic and various operators and strategies for route improvement. Calculation results show an improvement in 44 instances of Solomon test data. Case study of postal service is also provided. In [13] a two phase method using GA and random search incorporating SA is implemented to solve VRPTW in various scenarios. A two stage ant colony optimization algorithm is proposed in [14]. The proposed two stage of algorithm have different goals and both are independent of each other. The objective of first goal is to minimize the number of vehicles and in the second stage the total distance is minimized using local search methods. In [15] a hybrid algorithm Gen SAT is proposed. In this algorithm initial solution is obtained using push forward insertion heuristic. $\lambda$-Interchange mechanism is proposed which moves customers between routes to generate neighborhood solutions for the VRPTW. Simulated annealing and tabu search is used for searching of $\lambda$ - interchange. A hybridization of SA and ACO (IACS-SA) is proposed for solving VRPTW in [16]. The proposed algorithm combines the strength of both search heuristics. Moreover a new route construction rule, a new pheromone update rule and diverse local search approaches were proposed. In [17, 18] an enhanced ant colony system for solving VRPTW is applied in which other factors like urgency to serve, bias and wait factors were incorporated with distance in state transition rule.

Table 1. Approaches used in VRPTW

\begin{tabular}{|l|l|l|l|}
\hline Authors' & Approach & $\begin{array}{l}\text { Initial Solution/Solution } \\
\text { Construction }\end{array}$ & $\begin{array}{l}\text { Solution } \\
\text { Improvement/Operators }\end{array}$ \\
\hline Ming yoa, QI et al.[7] & VNS and TS & Solomon I1 insertion & $\begin{array}{l}\text { Exchange, opt and Cross } \\
\text { Exchange }\end{array}$ \\
\hline H.Nazif, L.S.Lee[8] & GA & Random & Swap and insertion \\
\hline Suraya Masorm[9] & GA and PSO & - & - \\
\hline $\begin{array}{l}\text { Bhawna Minocha and } \\
\text { Saswati Tripathi[13] }\end{array}$ & GA and Random Search & $\begin{array}{l}\text { Push Forward Insertion } \\
\text { Heuristic }\end{array}$ & SA \\
\hline Xudong Wu[14] & ACO & Solomon I1 insertion & Local Search \\
\hline $\begin{array}{l}\text { Sam R. Thangiah et al } \\
{[15]}\end{array}$ & Hybrid (GA, SA,TS) & $\begin{array}{l}\text { Push Forward Insertion } \\
\text { Heuristic }\end{array}$ & $\lambda$-interchange \\
\hline $\begin{array}{l}\text { Chea-Ho Chen and } \\
\text { ching-Jung Ting [16] }\end{array}$ & Hybrid (ACS and SA) & Nearest Neighbor & Local Search \\
\hline $\begin{array}{l}\text { Sandhya and V.Katiyar } \\
{[17,18]}\end{array}$ & ACS & Nearest Neighbor & - \\
\hline R. Banos et al.[21] & EA and SA & - & - \\
\hline $\begin{array}{l}\text { Phuong Khanh Nguyen } \\
{[22]}\end{array}$ & Hybrid & Solomon I1 insertion & Local Search \\
\hline
\end{tabular}


Pheromone update is also done with a minimum and maximum upper bound. An overview along with recent advances in ACO is discussed in [19]. In paper they also discuss the extensions, applications of ACO and various issues related to ACO. A multi objective evolutionary algorithm (MOEA) that can minimize any number of objectives and a mechanism for more diversity is proposed in which 1st offspring is selected as usual in EA but other offspring's are selected from non-common areas of the search space from the second parent [20]. A multi start evolutionary algorithm with $\mathrm{SA}$ is designed for solving VRPTW in [21] and tested on 100 customers of Solomon data set. In this paper populations are optimized using the mutation operators of usual EA and offspring's individually are accepted or rejected by applying SA. Vidal et al. [22] developed a hybrid GA with adaptive diversity management for VRPTW and it was claimed to be the best meta heuristic. Proposed method combines the exploration of GA and local search for intensification. Permutation encoding without deliminators is used for individual representation. Finally a split procedure is used to partition solution for getting final tour. A general tool using tabu search is proposed for solving VRPTW in [23]. In their work the neighborhood structure is exponential in size resulting in evaluation procedure of polynomial complexity. Summarization of approaches used in VRPTW is given in Table 1.

\section{METAHEURISTICS FOR VRPTW}

For solving optimization problem like VRPTW various methods are proposed in literature. Solving methods for VRPTW can be divided into 2 main categories as shown in Fig. 2

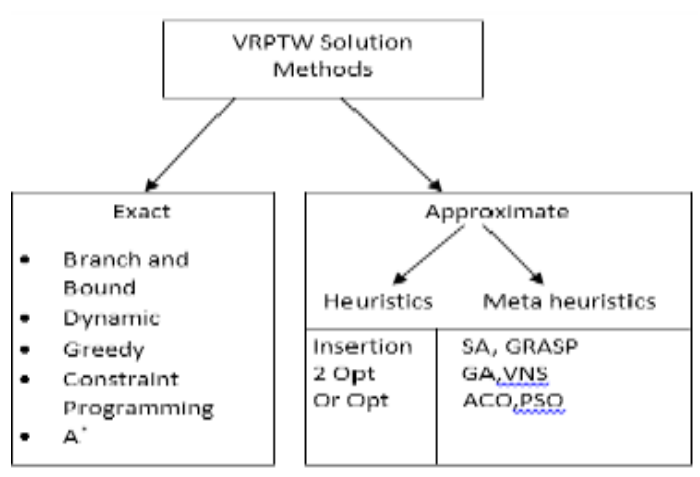

Fig. 2. Solution Methods for VRPTW problem

Exact methods guarantees to find optimal solution if sufficient time and space is given. On the other hand heuristics are the techniques that can find a feasible solution with reasonable time. Heuristic does not guarantee about the solution quality although it can be made by empirical analysis. A popular class of approximate method is meta heuristics that are the general frame work for the heuristic methods. Meta heuristics provides acceptable solution within a reasonable time by iteratively improving the candidate solution. The design space of meta heuristic is based on two contradictory criteria's: Intensification (exploitation) and diversification (exploration). Balance between these 2 criteria's is important for obtaining high quality solution by exploring all promising and non-promising areas and faster solution by exploring only promising areas. Various classification criteria's can be used for classifying meta heuristics:

1. Nature vs Non nature: Nature is the rich source of inspiration for many meta heuristics. Therefore almost all are nature inspired. Some of them are bio-inspired like GA, EA. Some are swarm inspired like ACO, PSO and other are from physics/chemistry based like SA. TS and DE are the example of former category.

2. Memory vs memory less: Some meta heuristics which uses memory for information extraction during search history are memory based meta heuristics. Some popular examples are ACO, Tabu etc. On the other hand some SA, GA are memory less meta heuristics.

3. Deterministic vs Stochastic: Stochastic meta heuristic solves optimization problems by applying random rules during their search process. The idea behind is to escape from local optima. On the other hand deterministic meta heuristics apply deterministic decisions to get some final solution by using the same initial solution.

4. Population based vs Single solution based: Another important characteristic for classification of meta heuristic is the number of solution used at the same time. Single solution based meta heuristic like SA and local search manipulate a single solution while in population based meta heuristics a whole set of solution is evolved. Some examples of this category are ACO, GA.

5. Trajectory vs multiple neighborhood moves: In trajectory method single neighborhood structure exists and it allows worse move to accept whereas in case of multiple neighborhood moves meta heuristics use a set of neighborhood structure.

6. Attraction vs Non-attraction: In case of attraction based meta heuristics interaction of multiple agents takes place like in ACO. GA is the example of non- attraction meta heuristics.

7. Rule vs Equation based: In rule base explicit updation of rules are used like GA.

Table 2. Categorization of Meta heuristics on various parameters

\begin{tabular}{|l|l|l|l|l|l|}
\hline Features & SA & TS & GA & ACO & GRASP \\
\hline Trajectory & Y & Y & N & N & Y \\
\hline Population & N & N & Y & Y & N \\
\hline Memory & N & Y & P & Y & N \\
\hline $\begin{array}{l}\text { Multiple } \\
\text { Neighborhood }\end{array}$ & N & N & P & N & Y \\
\hline Dynamic & N & P & N & N & N \\
\hline Nature Inspired & Y & N & Y & Y & N \\
\hline Stochastic & Y & N & Y & Y & N \\
\hline
\end{tabular}


On these parameters various popular meta heuristics along with their properties are summarized in Table 2 . Here $\mathrm{Y}$ denotes the presence of feature in the meta heuristic, $\mathrm{N}$ represent the absence of feature and $\mathrm{P}$ represents the partial presence of feature.

Among these SA, GA and ACO were chosen for comparison keeping in mind that all three are nature inspired and stochastic in nature. On the other hand GA and ACO possess some common properties that are different from SA like both are population based meta heuristics and uses memory for information extraction. A brief overview of these meta heuristic are given below in
Table 4.

\section{XPERIMENTAL RESULTS FOR VRPTW}

In this paper we implemented SA, ACO and GA for solving VRPTW. Pseudo code for these three methods can be reviewed from [24]. These pseudo codes are implemented in MATLAB and executed on Windows 7 platform. After sensitive analysis values of various parameters used for these meta heuristics are given in Table 3.

Table 3. Value for various parameters

\begin{tabular}{|l|l|l|l|l|l|l|l|l|l|}
\hline Parameters & Population & $\begin{array}{l}\text { Mutatio } \\
\text { n Rate } \\
\text { Meta } \\
\text { heuristics }\end{array}$ & $\begin{array}{l}\text { Temp } \\
.\end{array}$ & $\begin{array}{l}\text { Coolin } \\
\text { g Rate }\end{array}$ & $\begin{array}{l}\text { Absolut } \\
\text { e Temp. }\end{array}$ & $\alpha$ & $\beta$ & $\gamma$ & $\rho$ \\
\hline GA & 1000 & 0.1 & & & & & & & \\
\hline SA & & & 10000 & 0.999 & 0.001 & & & \\
\hline ACO & & & & & & 1 & 3 & 1 & 0.2 \\
\hline
\end{tabular}

\section{A. Performance Metrics}

For empirical analysis between implemented meta heuristics various factors were taken, their detail description are as follows:

(a) Data set: In this paper 2 categories of problems are taken on the basis of time windows type 1 and type 2 . Type 1 problems have short time windows whereas type 2 problems have long time windows. Further these 2 categories consist of three problems based on geographical distribution of customers namely Random(R), Clustered (C) and Random Clustered (RC). The geographical distributions of 100 customers for different problems are shown in Fig 3 (a) and Fig 3 (b). Here circle nodes represent the customers and depot is shown by square. The layout for $\mathrm{C}$ and $\mathrm{RC}$ problems are same i.e $\mathrm{X}$ ranges from [-40, 60] and $\mathrm{Y}$ ranges from $[-50,40]$ and for $\mathrm{R}$ problems range for $\mathrm{X}$ is $[-40,40]$ and $\mathrm{Y}$ ranges from [40,50]. Fig 3 (a) shows clear clusters of customers having 10 customers per cluster and Fig 3 (b) shows the random distribution of customers and similar picture can be obtained for random clustered problem in which clusters are formed at the boundaries and random distribution of customers at the center.

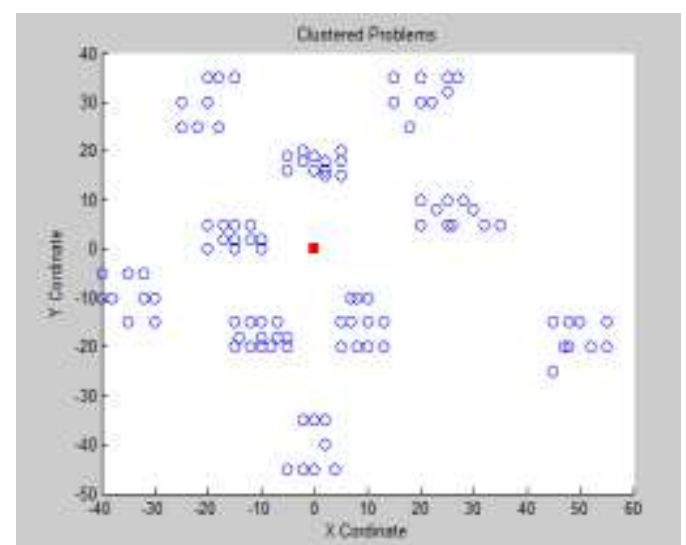

(a) C class problem

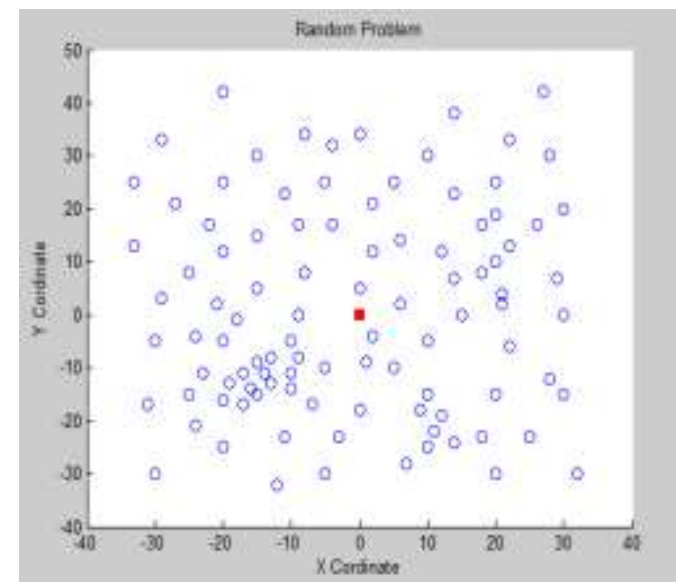

(b) $\mathrm{R}$ class problem

Fig. 3. (a), (b) Geographical Distribution of customers. 
Table 4. Features of SA, GA, ACO

\begin{tabular}{|c|c|c|c|}
\hline Features & $\mathrm{ACO}$ & GA & SA \\
\hline Proposed By & Marco Dorigo & John Holland & Kirkpatrick, Gelatt and Vecchi \\
\hline Inspiration Source & $\begin{array}{l}\text { Food Searching Behavior of } \\
\text { Ants }\end{array}$ & Evolution and natural Selection & Annealing in metallurgy \\
\hline Working & $\begin{array}{l}\text { 1. Initialize parameters. } \\
\text { 2. Repeat step } 3 \text { and } 4 \text { until } \\
\text { stopping condition is met. } \\
\text { 3. Construct Solutions } \\
\text { 4. Update pheromone trails } \\
\text { 5. Output result. }\end{array}$ & $\begin{array}{l}\text { 1. Generate populations and fitness } \\
\text { function. } \\
\text { 2. Evaluate population. } \\
\text { 3. Repeat following until stopping } \\
\text { condition is met. } \\
\text { (a) Crossover } \\
\text { (b) Mutation } \\
\text { (c) Evaluate } \\
\text { 4. Output result. }\end{array}$ & $\begin{array}{l}\text { 1. Initialize temperature and } \\
\text { generate initial solution. } \\
\text { 2. Repeat step } 3 \text { and } 4 \text { until stopping } \\
\text { condition is met. } \\
\text { 3. Generate new solution by making } \\
\text { change to current solution. } \\
\text { 4. Accept solution with some } \\
\text { probability. } \\
\text { 5. Output result. }\end{array}$ \\
\hline Advantages & $\begin{array}{l}\text { 1. Distributed Computing } \\
\text { 2. Performance does not relies on } \\
\text { initial solution. } \\
\text { 3. Retains knowledge of good } \\
\text { solution. } \\
\text { 4. Robust and can be hybridized } \\
\text { with other algorithms. } \\
\text { 5. Best suited for problems which } \\
\text { are dynamic in nature. }\end{array}$ & $\begin{array}{l}\text { 1. Performance does not relies on initial } \\
\text { solution. } \\
\text { 2. Best solution but high time } \\
\text { computation. } \\
\text { 3. Applicable for multiobjective problems. } \\
\text { 4. Best suited for problems having no } \\
\text { mathematical analysis. }\end{array}$ & $\begin{array}{l}\text { 1. Easy to code for complex problems. } \\
\text { 2. Can handle problems having } \\
\text { unordered data and many } \\
\text { constraints. } \\
\text { 3. Statically guarantees to find global } \\
\text { optimal solution. }\end{array}$ \\
\hline Disadvantages & $\begin{array}{l}\text { 1. Pheromone stagnation results in } \\
\text { premature convergence. } \\
\text { 2. Ant communication mechanism } \\
\text { is time consuming. }\end{array}$ & $\begin{array}{l}\text { 1. Knowledge of good solutions can be } \\
\text { destroyed by operators. } \\
\text { 2. Difficult to apply for dynamic and highly } \\
\text { constraint problems. }\end{array}$ & $\begin{array}{l}\text { 1. Performance relies on initial } \\
\text { solution. } \\
\text { 2. Continuous annealing is time } \\
\text { consuming. } \\
\text { 3. Tradeoff between solution quality } \\
\text { and time consumption. }\end{array}$ \\
\hline
\end{tabular}

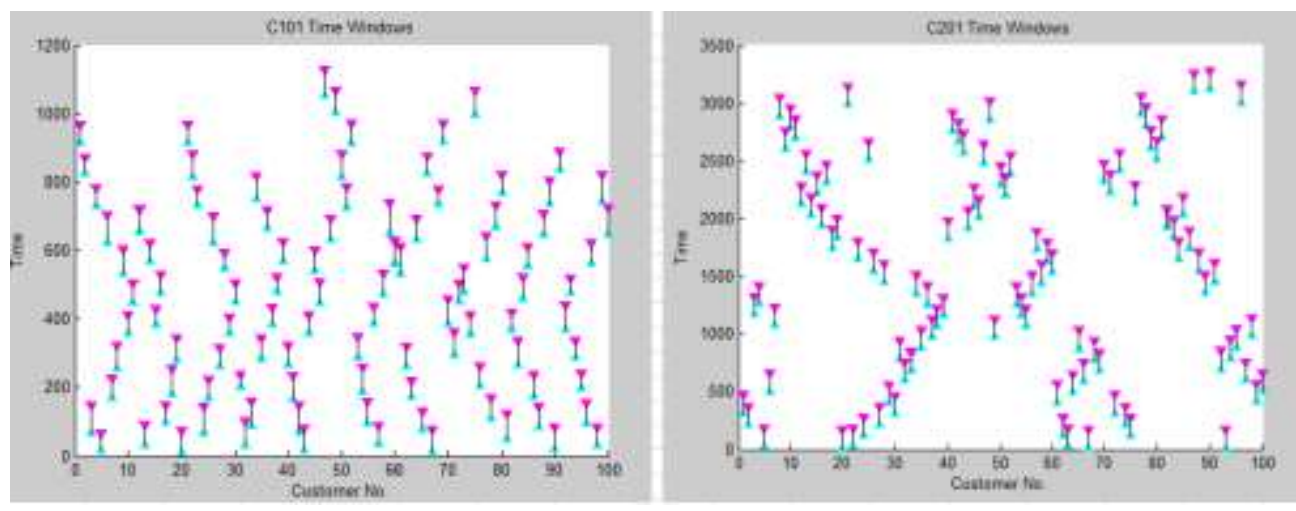

Fig. 4. (a) and (b)

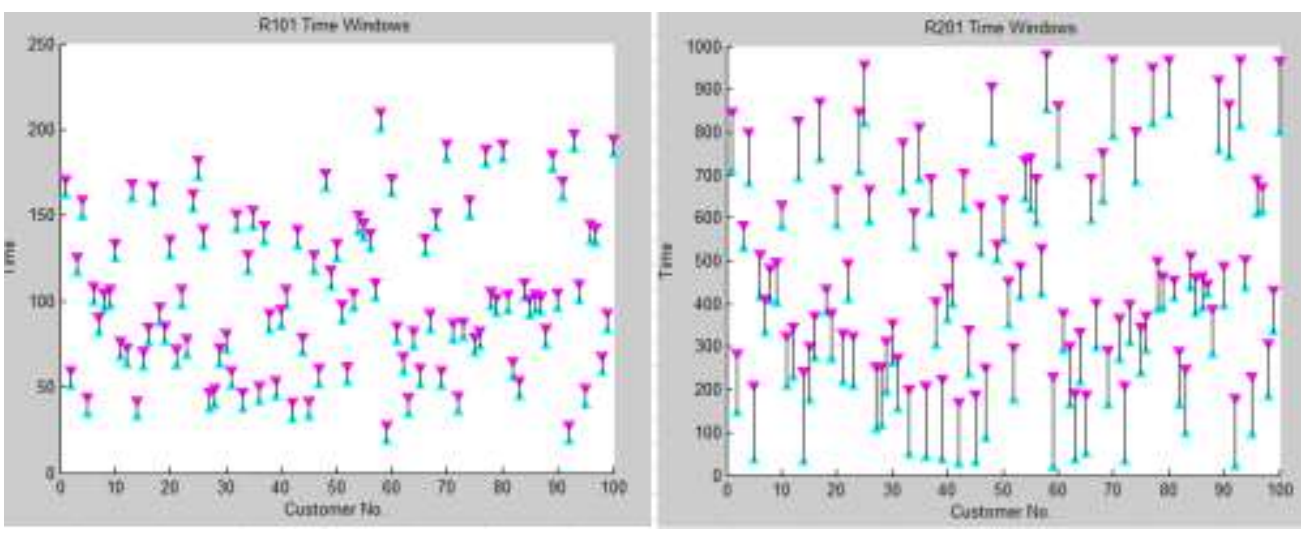

Fig. 4. (c) and (d) 


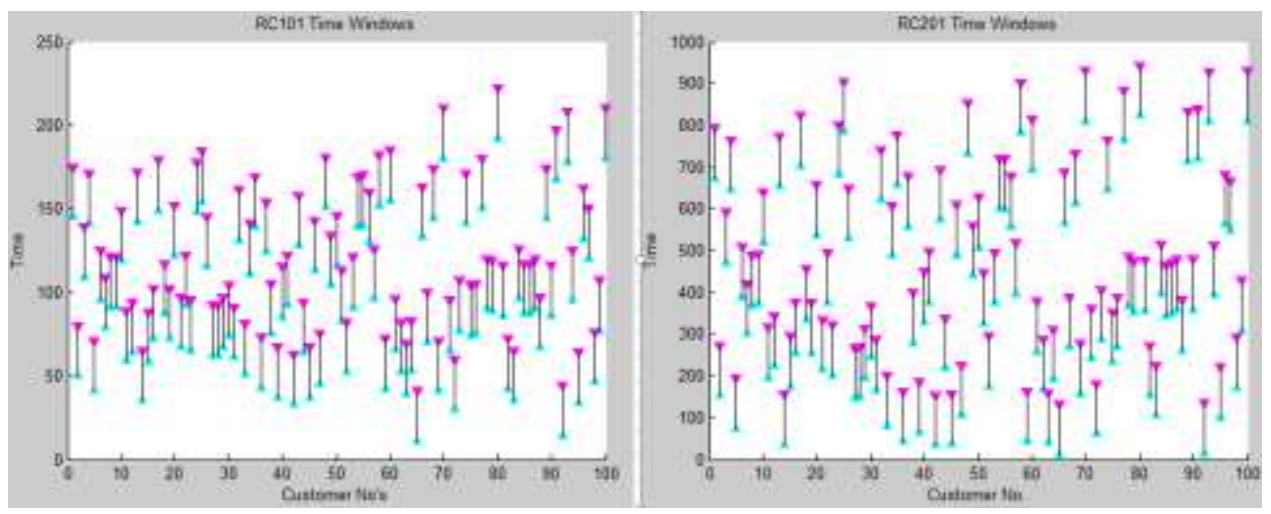

Fig. 4. (e) and (f)

Fig 4 (a) and (b) represents the characteristic of time windows for $\mathrm{C} 1$ problem and $\mathrm{C} 2$ problem. Fig 4 (c) and (d) represents the characteristics of time windows for R1 and R2 problem while fig 4 (e) and (f) shows for RC1 and $\mathrm{RC} 2$ problems. In all three cases $\mathrm{X}$ axis represents the customer number and $\mathrm{Y}$ axis denotes the time. Vertical bar represents the time windows.

Table 5 shows the results obtained for problems with 25, 50 and 100 customers respectively.

Table 5. Total travelled distance and CPU time for 25, 50, 100 customers

\begin{tabular}{|c|c|c|c|c|c|c|c|c|c|}
\hline $\begin{array}{r}\text { No. of } \\
\text { Comers }\end{array}$ & & Initial & lution & & $\mathbf{A}$ & & GA & & $\mathrm{CO}$ \\
\hline \multirow{7}{*}{25} & Problem & TD & $\begin{array}{l}\text { Time } \\
\text { (sec) }\end{array}$ & TD & $\begin{array}{l}\text { Time } \\
\text { (sec) }\end{array}$ & TD & $\begin{array}{l}\text { Time } \\
\text { (sec) }\end{array}$ & TD & $\begin{array}{l}\text { Time } \\
\text { (sec) }\end{array}$ \\
\hline & R1 & 2464.7 & .5 & 451.5 & 71 & 429.6 & 210 & 427.8 & 137 \\
\hline & C1 & 2459.1 & .3 & 198.2 & 484.6 & 191.3 & 187.7 & 191.3 & 115 \\
\hline & RC1 & 3419.1 & .3 & 342.3 & 67.6 & 351.5 & 193.8 & 342.3 & 56 \\
\hline & $\mathbf{R 2}$ & 3384.7 & .3 & 456.5 & 56 & 417 & 365.2 & 417 & 71 \\
\hline & $\mathrm{C2}$ & 2140.3 & .3 & 461 & 164 & 410 & 368 & 410 & 267 \\
\hline & RC2 & 4251.6 & .3 & 315.7 & 58 & 301.5 & 226.1 & 301.5 & 54 \\
\hline \multirow{6}{*}{50} & R1 & 2648.5 & 1 & 772.2 & 367.2 & 694.7 & 1028 & 696.3 & 872 \\
\hline & C1 & 2483.0 & 1 & 401.2 & 484.6 & 398.7 & 936.1 & 327 & 295 \\
\hline & RC1 & 3523.8 & 1 & 685.95 & 150.3 & 669.2 & 806.4 & 677.8 & 121 \\
\hline & $\mathbf{R 2}$ & 3604.9 & 1 & 639.1 & 258 & 581.5 & 1544.14 & 573.7 & 783 \\
\hline & $\mathrm{C2}$ & 2363.6 & 1 & 717.4 & 993.5 & 751.3 & 1549.7 & 720.1 & 873 \\
\hline & RC2 & 4495.3 & 1 & 665.3 & 554.8 & 588 & 2239.7 & 587.7 & 623 \\
\hline \multirow{6}{*}{100} & R1 & 2550.6 & 7 & 1215.8 & 2433.5 & 1198 & 2962 & 1201.7 & 2347 \\
\hline & C1 & 2310.0 & 6 & 968.7 & 2461.3 & 873.5 & 2993.3 & 828.9 & 582 \\
\hline & RC1 & 3307.6 & 6 & 1442.7 & 1132.4 & 1308.5 & 3550.2 & 1308.5 & 2107 \\
\hline & R2 & 3592.8 & 6 & 962.2 & 2100 & 1154 & 5206.4 & 962.2 & 2154 \\
\hline & $\mathrm{C2}$ & 3572.3 & 6 & 1261.6 & 4447.5 & 1362.4 & 6114 & 1261.6 & 4232 \\
\hline & RC2 & 4778.3 & 6 & 1165 & 2375.4 & 1138 & 8875 & 1143.9 & 2884 \\
\hline
\end{tabular}

(b) Improvement in total distance: Improvement in total travel distance means how much results are optimized after applying meta heuristics. In this paper initial solution is obtained by nearest neighbor and saving is calculated as follows and is summarized for three metaheuristics for 100 customers in table 6 .

$$
\text { Improvement }=\frac{T D_{N N}-T D_{\text {Metaheuristic }}}{T D_{N N}} * 100
$$

Table 6. Improvement in total distance for 25, 50, 100 customers

\begin{tabular}{|c|c|c|c|c|c|c|c|c|c|c|c|c|c|c|c|c|c|c|}
\hline Problem & \multicolumn{3}{|l|}{ R1 } & \multicolumn{3}{|l|}{ C1 } & \multicolumn{3}{|c|}{ RC1 } & \multicolumn{3}{|l|}{ R2 } & \multicolumn{3}{|l|}{ C2 } & \multicolumn{3}{|c|}{ RC2 } \\
\hline $\begin{array}{l}\text { No. of } \\
\text { Customers }\end{array}$ & 25 & 50 & 100 & 25 & 50 & 100 & 25 & 50 & 100 & 25 & 50 & 100 & 25 & 50 & 100 & 25 & 50 & 100 \\
\hline $\begin{array}{l}\text { Meta } \\
\text { heuristics }\end{array}$ & $\%$ & прг & emen & & & & & & & & & & & & & & & \\
\hline SA & 82 & 71 & 52 & 92 & 84 & 58 & 90 & 81 & 56 & 87 & 82 & 73 & 78 & 70 & 65 & 93 & 85 & 76 \\
\hline GA & 83 & 74 & 53 & 92 & 84 & 62 & 90 & 81 & 60 & 88 & 84 & 68 & 81 & 68 & 62 & 93 & 87 & 76 \\
\hline ACO & 83 & 74 & 53 & 92 & 87 & 64 & 90 & 81 & 60 & 88 & 84 & 73 & 81 & 70 & 65 & 93 & 87 & 76 \\
\hline
\end{tabular}


It can be obtained from the above table that the $\%$ of improvement is high for small size problems and it worsen as the problem size increases because the number of possible permutation increases exponentially. All meta heuristics performs well for different problems but the \% of improvement is high for ACO when compared with SA and GA.

(c) Time Complexity: Time complexity of the algorithm is calculated in terms of CPU time taken by the meta to run to its execution. In this paper time is measured in seconds and is shown in fig 5 (a)-(f) for different instances. Three observations can be made that (i) time complexity grows with the size of input and (ii) GA is the most time consuming meta heuristic (iii) SA gives good solutions in faster run time.

(d) Solution quality: Solution quality is measured in terms of total travel distance. Less distance represents the high quality solution while high travel distance means comparatively less quality solution. From fig 6 (a)-(f) it can be concluded that solution quality is good for small size problems but it reduces as the size increases. For large size problems solution quality provided by GA is better that SA and ACO.
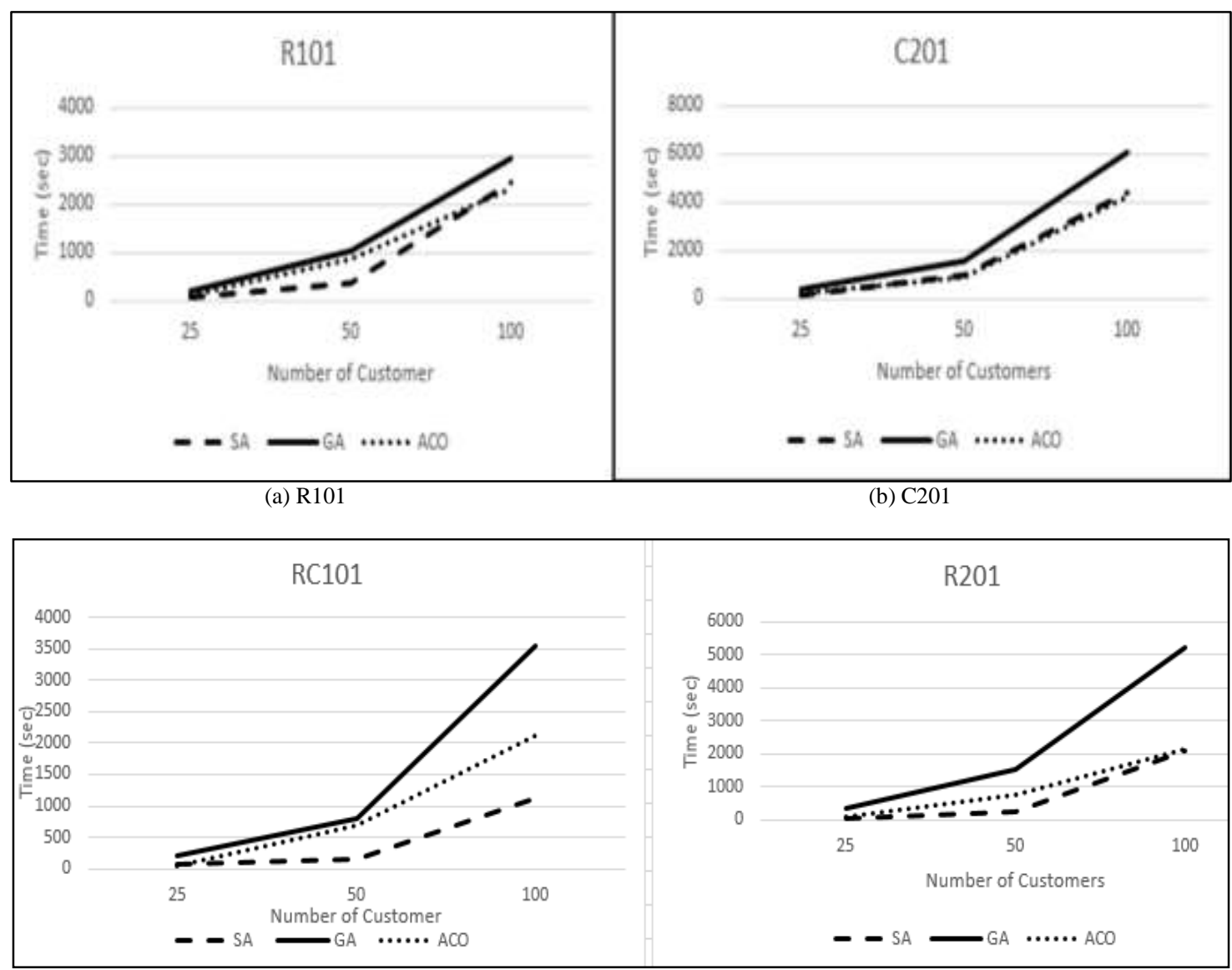

(c) $\mathrm{RC} 101$

(d) R201
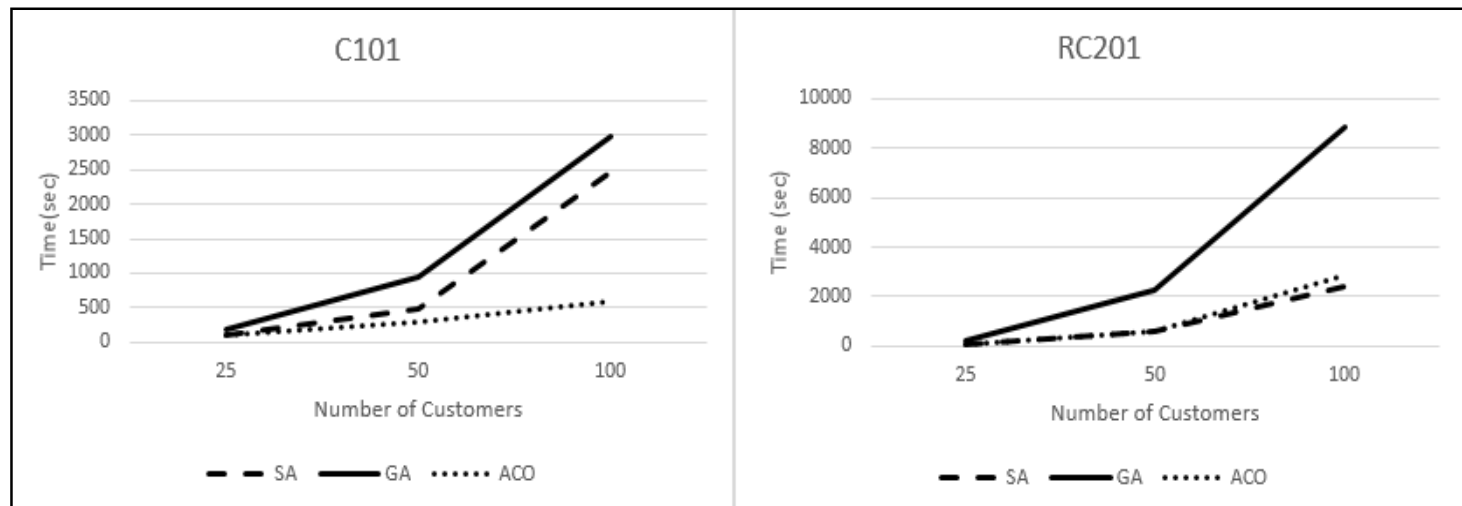

(e) $\mathrm{C} 101$

(f) RC201

Fig. 5. Time complexity for instance 
(e) Robustness: Robustness means how the performance of meta heuristics changes if some input parameter is changed. A robust algorithm should perform consistently over wide variety of problems and should not be sensitive to changes in input size. In this paper numbers of customers are varied from 25,50 and 100 to check the robustness of meta heuristics. It can be observed from table 4.2 (a)-(c) that ACO and GA provides robust results for all three cases but the robustness for SA degrades with the increase in number of customers. (f) Simplicity: Simplicity refers to ease of coding. Ease of implementation means the code should be simple, short, self-contained, effective neighborhood moves and has less parameter. In terms of simplicity SA is better than ACO and GA.

(g) Resource Consumption: Resource consumption means the memory space taken by meta heuristics. $\mathrm{SA}$ is better in terms of resource consumption when compared to GA and ACO as no intermediate results will be stored.

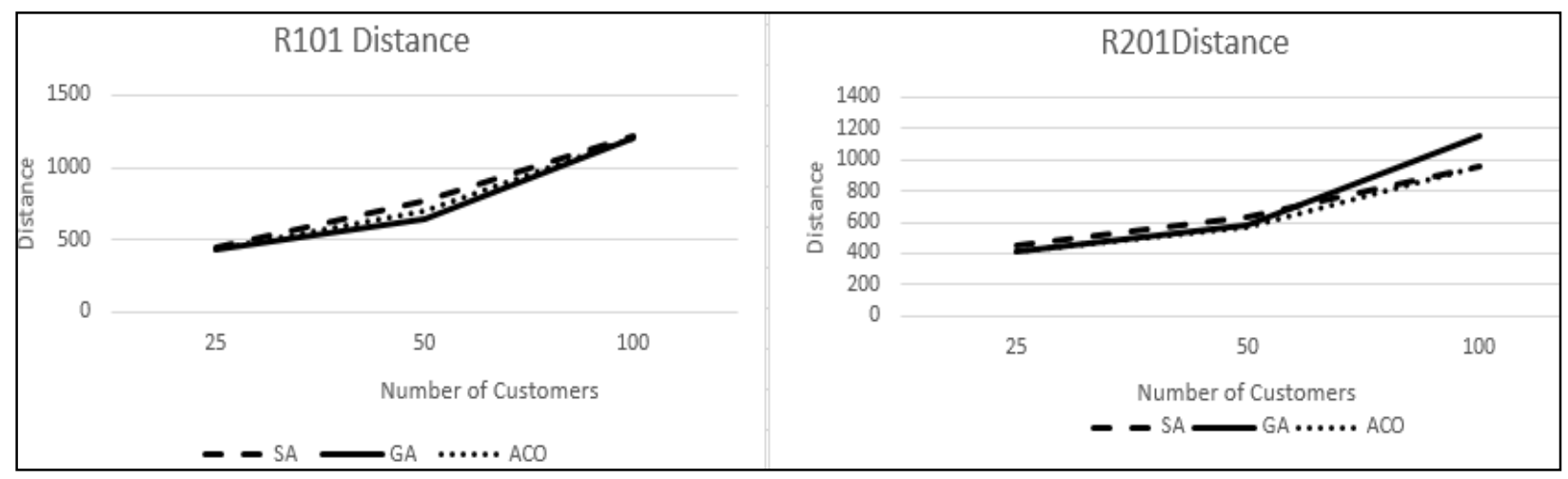

(a) R101

(b) R201

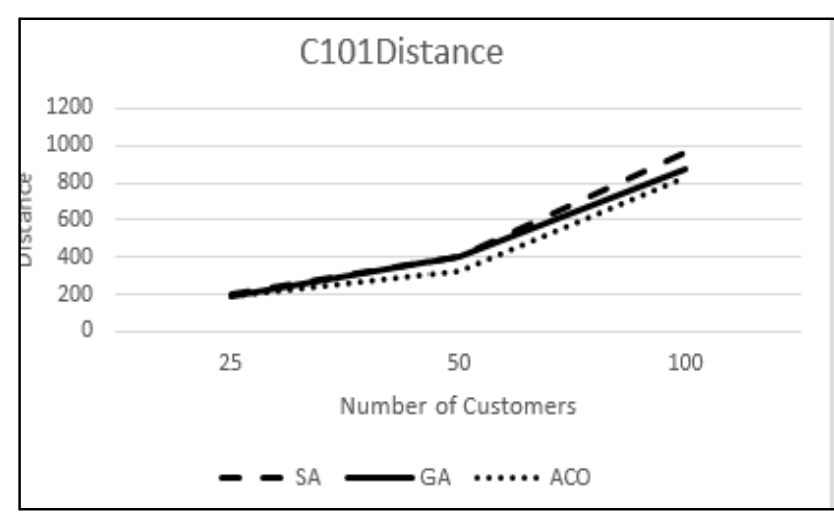

(c) $\mathrm{C} 101$

(e) $\mathrm{RC} 101$

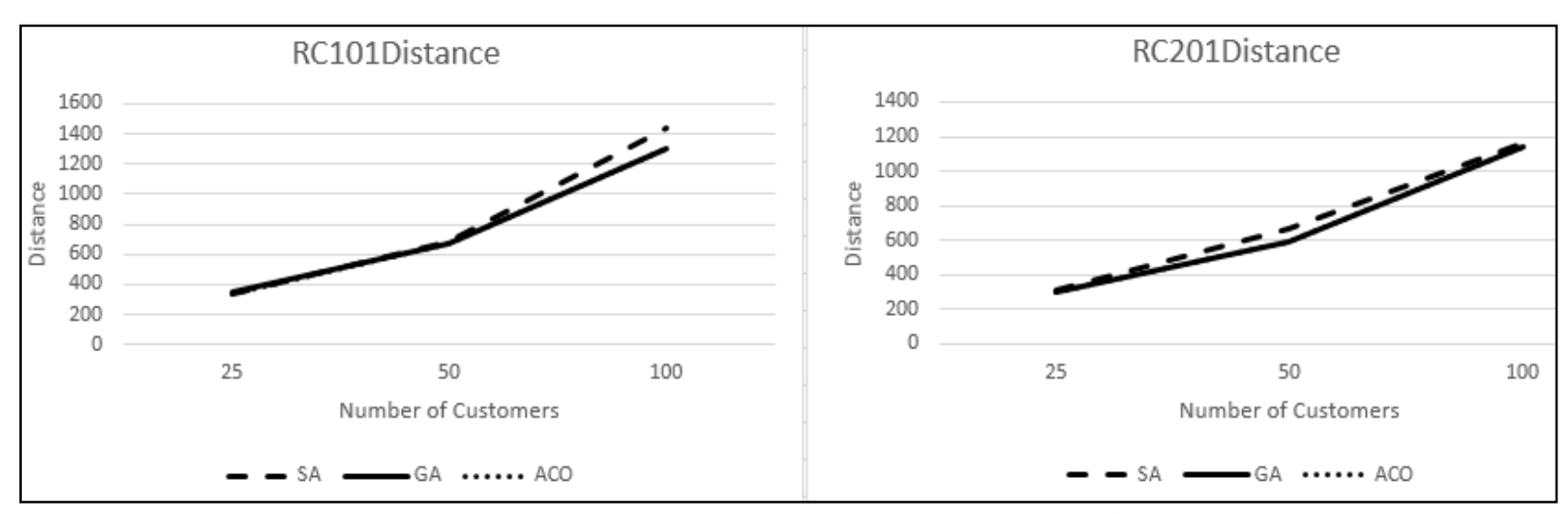

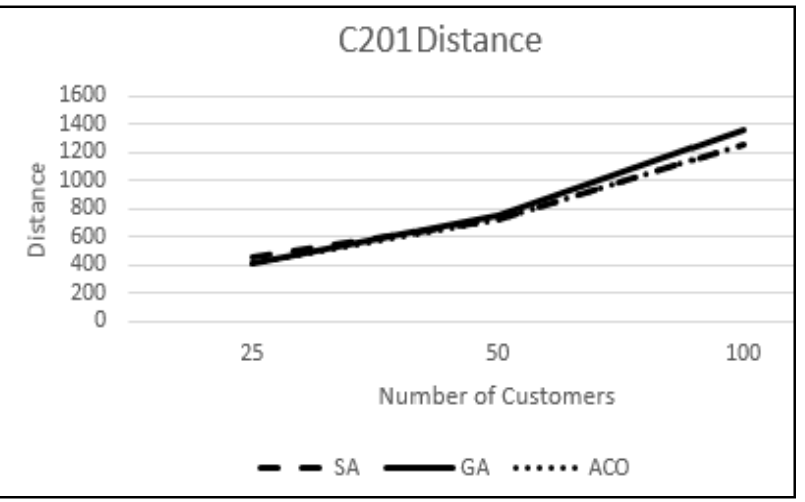

(d) C201

Fig. 6. Solution quality for instance

Table 7 summarizes the findings of our analysis about SA, GA and ACO. In this table $\mathrm{H}$ stands for high, $\mathrm{M}$ stands for medium and $\mathrm{L}$ stands for low presence of particular attribute. From the table it can be concluded that SA is simple to code but its presence is low on all other attributes discussed above. On the other hand both GA and ACO even complex to code but produces high quality results. 
Table 7. Comparative analysis of meta heuristics

\begin{tabular}{|c|c|c|c|c|c|c|c|c|}
\hline & & Dosast & Inpvizinert & $\begin{array}{l}\text { Tint } \\
\text { Conplesity }\end{array}$ & $\begin{array}{l}\text { Soldisiso } \\
\text { Quality }\end{array}$ & Robusters & Simplacty & \begin{tabular}{l|} 
Resucoce \\
Cossurfinan
\end{tabular} \\
\hline S. & VIH & L & N & $\overline{\mathrm{L}}$ & L & L & H & L \\
\hline$\overline{G H}$ & H & 甘 & $\mathbb{X I H}$ & 甘 & H & B & II & MI \\
\hline 100 & $\mathbb{H}$ & VIH & $\mathrm{H}$ & M & VII & E & XI & $\mathbb{H}$ \\
\hline
\end{tabular}

\section{CONCLUSION AND FUTURE WORK}

VRPTW is an important problem in transportation and it is a well-known NP hard combinatorial problem. Various metaheuristics are used for solving it. In this paper three widely used meta heuristics namely SA, GA and ACO are implemented and an empirical analysis of obtained optimal solution is compared on different parameters. The carried out analysis allows us to draw following conclusion: (1) Resources are expensive and optimization is necessary and meta heuristics are the good choice. (2) There is tradeoff between solution quality and time complexity. (3) SA gives good solution in faster run time. (4) Solution quality provided by ACO and GA is better than SA but the time complexity of GA is high and ACO lies between SA and GA. (5) In terms of robustness both ACO and GA scores high than SA but with high simplicity score. (6) GA is better choice for powerful systems because it provides good quality results with high time and resource consumption. (7) Results produced by ACO is better than GA and SA both in terms of solution quality and time although the differences are not overwhelming. At the end it can be concluded that the choice of meta heuristic depends upon various factors. The main objective is to make a good tradeoff between time and solution quality. In future these techniques can be hybridized with each other and with other method so that high quality results can be obtained with less time complexity.

\section{ACKNOWLEDGMENT}

The authors wish to thank Dr. Chander Mohan from Ambala College of Engineering, ambala, Haryana for his suggestions and corrections.

\section{REFERENCES}

[1] Gendreau, Michel, and Christos D. Tarantilis. Solving large-scale vehicle routing problems with time windows: The state-of-the-art. CIRRELT, 2010.

[2] Hosny, Manar Ibrahim. Investigating heuristic and metaheuristic algorithms for solving pickup and delivery problems. Diss. Cardiff University, 2010.

[3] Soo, Raymond Kuo Yang, and Yong Haur Tay. "A survey on the progress of research on vehicle routing problem with time window constraints." Symposium on Progress in Information and Communication Technology (SPICT).
2009.

[4] Eksioglu, Burak, Arif Volkan Vural, and Arnold Reisman. "The vehicle routing problem: A taxonomic review." Computers \& Industrial Engineering 57.4 (2009): 14721483.

[5] Blocho, Miroslaw, and Zbigniew J. Czech. "A parallel memetic algorithm for the vehicle routing problem with time windows."P2P, Parallel, Grid, Cloud and Internet Computing (3PGCIC), 2013 Eighth International Conference on. IEEE, 2013.

[6] Amini, Shahrzad, Hassan Javanshir, and Reza TavakkoliMoghaddam. "A PSO approach for solving VRPTW with real case study." Int. J. Res. Rev. Appl. Sci4.3 (2010): 118-126.

[7] QI, Mingyao, et al. "A new two-phase hybrid metaheuristic for vehicle routing problem with time windows." Journal of the Eastern Asia Society for Transportation Studies 10.0 (2013): 880-896.

[8] Nazif, Habibeh, and Lai Soon Lee. "Optimized crossover genetic algorithm for vehicle routing problem with time windows." American journal of applied sciences 7.1 (2010): 95.

[9] Masrom, Suraya, and Arfah Mohd Nasir. "Evaluation of vehicle routing problem with time windows by using metaheuristics algorithms." (2012).

[10] El-Sherbeny, Nasser A. "Vehicle routing with time windows: An overview of exact, heuristic and metaheuristic methods." Journal of King Saud UniversityScience 22.3 (2010): 123-131.

[11] Yang, Xin-She. "Metaheuristic Optimization: Algorithm Analysis and Open Problems." arXiv preprint arXiv: 1212 0220 (2012).

[12] Taner, Filip, Ante Galić, and Tonči Carić. "Solving Practical Vehicle Routing Problem with Time Windows Using Metaheuristic Algorithms." PROMETtraffic\&Transportation 24.4 (2012): 343-351.

[13] Minocha, Bhawna, and Saswati Tripathi. "Two Phase Algorithm for Solving VRPTW Problem." (2013).

[14] Wu, Xudong. "A two-stage ant colony optimization algorithm for the vehicle routing problem with time windows." IJACT: International Journal of Advancements in Computing Technology 4.1 (2012): 485-491.

[15] Thangiah, Sam R., Ibrahim H. Osman, and Tong Sun. "Hybrid genetic algorithm, simulated annealing and tabu search methods for vehicle routing problems with time windows." Computer Science Department, Slippery Rock University, Technical Report SRU CpSc-TR-94-27 69 (1994).

[16] Chen, Chia-Ho, and Ching-Jung Ting. "A hybrid ant colony system for vehicle routing problem with time windows." Journal of the Eastern Asia Society for Transportation Studies 6 (2005): 2822-2836.

[17] Bansal, Sandhya, Rajeev Goel, and C. Mohan. "Use of Ant Colony System in Solving Vehicle Routing Problem with Time Window Constraints." Proceedings of the Second International Conference on Soft Computing for Problem Solving (SocProS 2012), December 28-30, 2012. Springer India, 2014.

[18] Katiyar, Vijay. "An Enhanced Ant Colony System for Solving Vehicle Routing Problem with Time Window." International Journal of Computer Applications 73 (2013).

[19] Dorigo, Marco, and Thomas Stützle. "Ant colony optimization: overview and recent advances." Handbook of metaheuristics. Springer US, 2010. 227-263.

[20] Garcia-Najera, Abel, and John A. Bullinaria. "An improved multi-objective evolutionary algorithm for the vehicle routing problem with time windows."Computers 
\& Operations Research 38.1 (2011): 287-300.

[21] Baños, Raúl, et al. "A multi-start hybrid algorithm for vehicle routing problems with time windows." World Online Conference on Soft Computing in Industrial Applications. 2011.

[22] Nguyen, Khanh Phuong. "Meta-heuristic Solution Methods for Rich Vehicle Routing Problems." (2014).

[23] Moccia, Luigi, Jean-François Cordeau, and Gilbert Laporte. "An incremental tabu search heuristic for the generalized vehicle routing problem with time windows." Journal of the Operational Research Society 63.2 (2012): 232-244.

[24] Blum, Christian, and Andrea Roli. "Metaheuristics in combinatorial optimization: Overview and conceptual comparison." ACM Computing Surveys (CSUR) 35.3 (2003): 268-308.

[25] Carić, Tonči, et al. "Empirical analysis of two different metaheuristics for real-world vehicle routing problems." Hybrid Metaheuristics. Springer Berlin Heidelberg, 2007. 31-44.

[26] Singh, Harpreet, and Parminder Kaur. "Website Structure Optimization Model Based on Ant Colony System and Local Search." International Journal of Information Technology and Computer Science (IJITCS) 6.11 (2014): 48.

[27] Kumar, Koushal, and Gour Sundar Mitra Thakur. "Advanced applications of neural networks and artificial intelligence: A review." International Journal of Information Technology and Computer Science (IJTCS) 4.6 (2012): 57.

\section{Authors' Profiles}

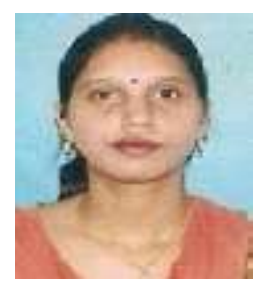

Sandhya is a research scholar at Maharishi Markandeswar University Mullana. She received his M.Tech degree from Banasthali Uniiversity, Jaipur (Rajasthan) and B.tech degree from Kurukshethra University (Haryana). She has supervised 8 M.Tech candidates. Her areas of interest are WSN, Meta heuristics and VRP. She has about 9 research papers in international journals of repute. Presently she is working at M.M.U as assistant professor.

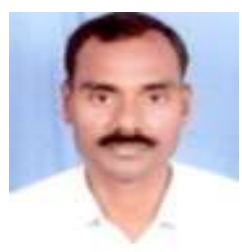

V. Katiyar born in Kanpur, India, on 30th June 1972. He received his Ph.D degree from M. M. University Mullana and B.E \& M.E. degrees from Kumaon University Nainital (U.P) and Thapar University Patiala (Punjab) respectively. He has supervised $25 \mathrm{M}$. Tech and $1 \mathrm{M}$. Phil candidates. His research interests are in Wireless Sensor Networks, Reliability Theory and Artificial Neural Networks, etc. He has about 18 years experience in teaching. He has published about 25 research papers in international journals of repute. Presently he is supervising $8 \mathrm{Ph}$. $\mathrm{D}$ and $8 \mathrm{M}$. Tech candidates.

How to cite this paper: Sandhya, V. Katiyar,"Relative Performance of Certain Meta Heuristics on Vehicle Routing Problem with Time Windows", International Journal of
Information Technology and Computer Science(IJITCS), vol.7, no.12, pp.40-49, 2015. DOI: 10.5815/ijitcs.2015.12.05 